\title{
Entangled Brachistochrone: Minimum Time to reach Target Entangled State
}

\author{
Arun K. Pati, Biswajit Pradhan and Pankaj Agrawal \\ Institute of Physics, Bhubaneswar-751005, Orissa, India
}

(Dated: November 10, 2018)

\begin{abstract}
We address the question: Given an arbitrary initial state and a general physical interaction what is the minimum time for reaching a target entangled state? We show that the minimum time is inversely proportional to the quantum mechanical uncertainty in the non-local Hamiltonian. We find that the presence of initial entanglement helps to minimize the waiting time. Furthermore, we find that in a bi-local rotating frame the entangling capability is actually a geometric quantity. We give an universal bound for the time average of entanglement rate for general quantum systems. The time average of entanglement rate does not depend on the particular Hamiltonian, rather on the fluctuation in the Hamiltonian. There can be infinite number of nonlocal Hamiltonians which may give same average entanglement rate. We also prove a composition law for minimum time when the system evolves under a composite Hamiltonian.
\end{abstract}




\section{INTRODUCTION}

Quantum entanglement plays a pivotal role in the emerging field of quantum information theory [1]. Creation, storage and processing of entangled states are challenging experimental tasks in any quantum information processing devices [2, 3, 4]. In recent years there have been considerable progress in understanding various types of entangled states. However, in multiparticle situations, many questions are still unexplored. We know that if we start from an unentangled state of two (or more) particles and allow them to interact, then the state may evolve to an entangled state depending on the initial state and the type of interaction Hamiltonian. The Hamiltonian that is capable of creating entanglement is not sum of local Hamiltonians, rather it involves a nonlocal part. This is so, because sum of the local Hamiltonians will always give rise to tensor product of local unitary evolution operators and we know that the later cannot create any entanglement. In some situation, one would like to generate a particular entangled state for some specific quantum information processing task. For example, if one could create a maximally entangled state in a controlled way, then that can be used for quantum teleportation, dense coding, remote state preparation and so on. However, what type of initial state and what kind of non-local Hamiltonian can give the desired entangled state is far from clear. Also, it is not the case that immediately after switching on the interaction Hamiltonian we will have the target entangled state.

There has been considerable interest in the study of dynamics of entanglement. In particular, a pertinent question in this context is given a nonlocal Hamiltonian, how well one can create entangled states. This has been address by Dür et al [5]. They have introduced entangling capability of Hamiltonian for two qubits and also generalized for two qudits. It was found that it is better to start with initial entangled state and the best initial entanglement is independent of the physical process. Also, it was shown that one can improve the capability if we allow fast local operations and ancillas (in some cases) [5].

In this paper we address the following question: Given an arbitrary product state and a general physical interaction what is the minimum time for creating a target entangled state? This is precisely the entangled brachistochrone problem. This question is not only of fundamental interest but also of practical importance. The answer will be relevant in many experimental context where we want a fixed entangled state after having the interaction on. Because, that will allow us to generate the desired entangled state in a controlled way. This will also answer the question what is the minimum time an experimentalist needs to wait to create the target entangled state. In the course of our investigation, we show that the entanglement capability of non-local Hamiltonains is a geometric quantity. We prove a universal bound on the time average of the entanglement rate. Our result suggests that even though we cannot tell whether entangling rate and disentangling rates are same, but the maximum value of the time average of entanglement and disentanglement rates are the same.

We prove that the minimum time for creating a fixed entangled state not only depends on the initial state and final state, but also depends on the speed at which system evolves. The speed of the quantum evolution is governed by the fluctuation in the Hamiltonian $\Delta H$. In particular, we show that the minimum time required to reach a target entangled state depends inversely on the quantum mechanical uncertainty in the non-local Hamiltonian. We illustrate this for two qubits. Further, we will show that the time average of entanglement

rate is upper bounded by $2 \log N \frac{\Delta H}{\hbar S_{0}}$, where $N$ is the number of Schmidt coefficients in the entangled state, $\Delta H$ is the fluctuation in the nonlocal Hamiltonian and $S_{0}$ is the shortest 
geodesic connecting the initial and the final states. As an application of this bound we estimate what could be the ultimate entanglement rate for non-local Hamiltonians.

The present paper is organized as follows. In Sec. II, we briefly discuss the geometric uncertainty relation and the bound for the minimum time for entangled states. In Sec. III, we illustrate the bound for two qubits. We find that initial entanglement can help to reduce the waiting time in reaching a maximal entangled state. In Sec. IV, we show that entanglement rate in a bilocal rotating frame is actually related to the quantum fluctuation in the nonlocal Hamiltonian (the speed of the quantum evolution). Also, we provide a universal bound for the time average of the entanglement rate. In Sec. V, we discuss the question of minimum time when the system is driven by composite Hamiltonian. Finally, we conclude our paper in Sec. VI.

\section{GEOMETRIC UNCERTAINTY RELATION AND MINIMUM TIME}

Here, we consider bipartite systems but some of the results will be valid for more general situations. Whenever the result holds only for bipartite systems, we will say so. Consider an arbitrary initial product state $|\Psi(0)\rangle=|\psi(0)\rangle \otimes|\phi(0)\rangle$ of a bipartite composite system. The nonlocal Hamiltonian for the system is given by $H=H_{1} \otimes I+I \otimes H_{2}+H_{\text {int }}$. Under the action of the Hamiltonian the state evolves unitarily as $|\Psi(0)\rangle \rightarrow|\Psi(t)\rangle=\exp (-i H t / \hbar)|\Psi(0)\rangle$. Now, depending on the type of initial state and $H_{\text {int }}$ the resulting state will be entangled. Suppose, we evolve the system from $|\Psi(0)\rangle \rightarrow|\Psi(T)\rangle$ and our target entangled state is $\left|\Psi_{T}\right\rangle=|\Psi(T)\rangle$. Then, the question is what is the minimum time $T_{\min }$ for which we should evolve the combined system to get the desired state?

To answer this, we need some geometric ideas. Let $\{\Psi\}$ be a set of vectors in $\mathcal{H}=\mathcal{H}_{1} \otimes \mathcal{H}_{2}$. If these vectors are not normalized we can consider a set of vectors $\{\Psi /\|\Psi\|\}$ of norm one in $\mathcal{L}$. The set of rays of $\mathcal{H}$ is called the projective Hilbert space $\mathcal{P}\left(\mathcal{H}_{1} \otimes \mathcal{H}_{2}\right)$. If $\operatorname{dim} \mathcal{H}_{1}=N$ and $\operatorname{dim} \mathcal{H}_{2}=M$, then $\mathcal{H} \simeq \mathbf{C}^{N M}$. The projective Hilbert space is $\mathcal{P}=\left(\mathbf{C}^{N M}-\{0\}\right) / U(1)$ which is a complex manifold of dimension $(N M-1)$. This can also be considered as a real manifold of dimension $2(N M-1)$. Any quantum state at a given instant of time can be represented as a point in $\mathcal{P}$ via the projection map $\Pi:|\Psi\rangle \rightarrow|\Psi\rangle\langle\Psi|$. The evolution of the state vector can be represented by a curve $C: t \rightarrow|\Psi(t)\rangle$ in $\mathcal{H}$ whose projection $\Pi(C)$ lies in $\mathcal{P}$. Here, smooth mappings $C:[0, t] \rightarrow \mathcal{L}$ of an interval into a differentiable manifold are called smooth curves in the given manifold [7].

Now consider the unitary time evolution of a bipartite quantum system $|\Psi(0)\rangle \rightarrow|\Psi(t)\rangle=$ $\exp (-i H t / \hbar)|\Psi(0)\rangle$, where $H$ is the nonlocal Hamiltonian. If the state at later time is entangled, then using the Schmidt decomposition theorem we can write the combined state as

$$
|\Psi(t)\rangle=\sum_{n=1}^{N} \sqrt{\lambda_{n}(t)}\left|a_{n}(t)\right\rangle\left|b_{n}(t)\right\rangle,
$$

where $\lambda_{n}(t)$ 's are the Schmidt coefficients with $\sum_{n} \lambda_{n}(t)=1$ for all time; $\left|a_{n}(t)\right\rangle$ and $\left|b_{n}(t)\right\rangle$ are the orthonormal Schmidt basis. As the state evolves, it traces a path in the state space $\mathcal{P}$. There is a natural metric on $\mathcal{P}$ which is the Fubini-Study metric, defined as [6, 7, 8]

$$
d S^{2}=4\left(1-|\langle\Psi(t) \mid \Psi(t+d t)\rangle|^{2}\right)=4 \frac{\Delta H^{2}}{\hbar^{2}} d t^{2},
$$


where $\Delta H^{2}=\left\langle\Psi(t)\left|H^{2}\right| \Psi(t)\right\rangle-\langle\Psi(t)|H| \Psi(t)\rangle^{2}$ is the quantum mechanical uncertainty in the nonlocal Hamiltonian. For time-independent Hamiltonians $\Delta H$ is also time independent (because $H$ and $U(t)$ commutes). Using the notion of infinitesimal distance, one can define the rate or the speed $V$ at which the quantum system evolves in the projective Hilbert space. This is given by $V=\frac{d S}{d t}=2 \frac{\Delta H}{\hbar}$. We can write down an explicit expression for the speed as (with the initial state as the product state)

$$
V^{2}=\frac{4}{\hbar}\left[\Delta H_{1}^{2}+\Delta H_{2}^{2}+\Delta H_{\mathrm{int}}^{2}+C\left(H_{1}, H_{\mathrm{int}}\right)+C\left(H_{\mathrm{int}}, H_{1}\right)+C\left(H_{2}, H_{\mathrm{int}}\right)+C\left(H_{\mathrm{int}}, H_{2}\right)\right],
$$

where $\Delta H_{1}^{2}=\left\langle\psi(0)\left|H_{1}^{2}\right| \psi(0)\right\rangle-\left\langle\psi(0)\left|H_{1}\right| \psi(0)\right\rangle^{2}, \Delta H_{2}^{2}=\left\langle\phi(0)\left|H_{2}^{2}\right| \phi(0)\right\rangle-\left\langle\phi(0)\left|H_{2}\right| \phi(0)\right\rangle^{2}$, and $C(A, B)=\langle\Psi(0)|A B| \Psi(0)\rangle-\langle\Psi(0)|A| \Psi(0)\rangle\langle\Psi(0)|B| \Psi(0)\rangle$ being the correlation between $A$ and $B$ (here $A$ and $B$ can be $H_{1}, H_{2}$ or $H_{\text {int }}$ ).

We can also give an expression for the speed of the entangled state evolution using the Schmidt basis and coefficients. Now, using (1) the Fubini-Study entangled metric can be expressed as

$$
\begin{aligned}
d S^{2} & =\sum_{n} \lambda_{n}(t)\left(\left\langle\dot{a}_{n}(t) \mid \dot{a}_{n}(t)\right\rangle+\left\langle\dot{b}_{n}(t) \mid \dot{b}_{n}(t)\right\rangle\right) d t^{2} \\
& -\left[\sum_{n} \lambda_{n}(t)\left(i\left\langle a_{n}(t) \mid \dot{a}_{n}(t)\right\rangle+i\left\langle b_{n}(t) \mid \dot{b}_{n}(t)\right\rangle\right)\right]^{2} d t^{2} \\
& -2 \sum_{n m} \sqrt{\lambda_{n}(t) \lambda_{m}(t)}\left\langle a_{m}(t) \mid \dot{a}_{n}(t)\right\rangle\left\langle b_{m}(t) \mid \dot{b}_{n}(t)\right\rangle+\sum_{n} \frac{d \lambda_{n}(t)^{2}}{4 \lambda_{n}(t)} .
\end{aligned}
$$

This metric is $U(1)$ gauge invariant and is independent of the detailed dynamics of the systems. This is so, because, there can be many non-local Hamiltonians which may give the same path in $\mathcal{P}$. If the state is not entangled (one of the Schmidt number is one and others are zero), then $|\Psi(t)\rangle$ may be written as $|\Psi(t)\rangle=|a(t)\rangle|b(t)\rangle$ and the metric is then given by $d S^{2}=d S_{1}^{2}+d S_{2}^{2}$, where $d S_{i}^{2},(i=1,2)$ are the Fubini-Study metrics for the individual subsystems. In the above expression, the second and third terms represent the effect of entanglement on the metric structure.

Let us apply the nonlocal Hamiltonian for a time period $T$ and consider the quantum evolution $|\Psi(0)\rangle \rightarrow|\Psi(T)\rangle$. Suppose that the state $|\Psi(T)\rangle$ is our target state. The total distance travelled by the quantum system in going from the initial state to the target state (as measured by the Fubini-Study metric) is given by $S=2 \int_{\mathrm{C}} \frac{\Delta H}{\hbar} d t=2 \mathrm{Vt}$, where $V$ is given in (3). This is typically a longer path in the projective Hilbert space of the entangled system. Now, consider the shortest path $S_{0}$ between the initial and the target state. It is given by

$$
|\langle\Psi(0) \mid \Psi(T)\rangle|^{2}=\cos ^{2} \frac{S_{0}}{2} .
$$

Since the actual distance $S$ is greater than or equal to the shortest distance $S_{0}$ (the AnandanAharonov version of the uncertainty relation) we have

$$
\Delta H \quad T \geq \frac{\hbar S_{0}}{2}
$$


From the above geometric relation we have a bound on the time required to reach a target entangled state which is given by

$$
T \geq \frac{\hbar}{\Delta H} \cos ^{-1}|\langle\Psi(0) \mid \Psi(T)\rangle| .
$$

Therefore, to minimize the waiting time, we have to evolve the system faster and minimise the shortest path. However, if the initial and target states are fixed, then the waiting time can be reduced by evolving the system faster. It may be noted that the above equation is valid both for qubits and qudits as well as for multiparticle quantum systems.

\section{OPTIMAL TIME FOR TWO-QUBIT STATE}

In this section, we discuss the optimal time required to reach a two-qubit maximally entangled state starting from a pure product state and some partial entangled state. Further, we will illustrate how the presence of initial entanglement helps us to minimize the waiting time.

Let the initial state $|\Psi(0)\rangle$ of two qubits be

$$
\left|\Psi_{E}\right\rangle=\sqrt{p}|0\rangle|1\rangle+\sqrt{1-p}|1\rangle|0\rangle,
$$

where $0 \leq p \leq 1 / 2$. A general two qubit Hamiltonian can be written as

$$
H=\sum_{i=1}^{3} \alpha_{i} \sigma_{i} \otimes I+\sum_{j=1}^{3} \beta_{j} I \otimes \sigma_{j}+\sum_{i, j=1}^{3} \gamma_{i j} \sigma_{i} \otimes \sigma_{j}
$$

where $\alpha_{i}, \beta_{i}$ are real numbers and $\gamma$ is a real matrix and $\sigma$ 's are the usual Pauli matrices. Supplementing the evolution operator with local unitary operators, we can rewrite the Hamiltonian as $H=\sum_{k=1}^{3} \mu_{k} \sigma_{k} \otimes \sigma_{k}$. where $\mu_{1} \geq \mu_{2} \geq \mu_{3} \geq 0$ are the sorted singular values of the matrix $\gamma[5]$. Let $|\Psi(0)\rangle$ evolves under a general two qubit Hamiltonian. So the quantum evolution can be written as

$$
\left|\Psi_{E}\right\rangle \rightarrow\left|\Psi_{T}\right\rangle=e^{-i H T / \hbar}\left|\Psi_{E}\right\rangle=e^{-i T \sum_{k=1}^{3} \mu_{k} \sigma_{k} \otimes \sigma_{k}}\left|\Psi_{E}\right\rangle=\left|\Psi^{+}\right\rangle,
$$

where $\left|\Psi^{+}\right\rangle=\frac{1}{\sqrt{2}}(|0\rangle|1\rangle+|1\rangle|0\rangle)$ is a maximally entangled state. The speed with which two qubit state evolves in time is given by

$$
V=\frac{2 \Delta H}{\hbar}=\left(\mu_{1}+\mu_{2}\right) \sqrt{1-4 p(1-p)} .
$$

When the target is a maximally entangled state, then the bound on time is given by

$$
T \geq \frac{S_{0}}{2\left(\mu_{1}+\mu_{2}\right) \sqrt{1-4 p(1-p)}},
$$

where $S_{0}=2 \cos ^{-1}(\sqrt{p}+\sqrt{1-p}) / \sqrt{2}$. For the choice of best initial entangled state, we have $p=p_{0} \approx 0.0832[5]$. This state has entanglement $E\left(\Psi_{E}\right) \approx 0.413$ ebit. To increase 
the entanglement from 0.413 ebit to one ebit, we must wait for a minimum time given by $T_{\min }=\frac{0.5911}{\left(\mu_{1}+\mu_{2}\right)}$.

Next, we ask: does the presence of initial entanglement help to reduce the waiting time? The answer is indeed yes. Instead of an initial entangled state, if we start from a product state $|\Psi(0)\rangle=|0\rangle|1\rangle$ and apply the unitary operator $U(T)$ to reach a maximally entangled state (which is our target state), then the bound is given by $T \geq \frac{S_{0}}{\left(\mu_{1}+\mu_{2}\right)}$, where $S_{0}=$ $2 \cos ^{-1}\left(\left|\left\langle 01 \mid \Psi^{+}\right\rangle\right|\right)$The minimum time is given by $T_{\min }=\frac{0.7854}{\left(\mu_{1}+\mu_{2}\right)}$. This shows that if we start from a product state and want to reach a maximally entangled state, then we will have to wait longer. This also illustrates the role of initial condition (or initial entanglement) in the brachistocrone problem.

\section{BOUND ON TIME AVERAGE OF ENTANGLEMENT RATE}

The study of dynamics of entanglement in quantum systems is an ongoing area of research. Given a nonlocal Hamiltonian, it is important to know how well one can create entangled states. In Ref. [5], it was found that it is better to start with initial entangled state and best initial entanglement is independent of the physical process. In this section, we will show that, in a bilocal rotating frame, the entangling rate is in fact a geometric quantity.

To quantify the entanglement production, one can define the entanglement rate $\Gamma(t)=$ $\frac{d E(\Psi(t))}{d t}$, where $E(\Psi)$ is some entanglement measure for the state $|\Psi(t)\rangle$. If we consider von Neumann entropy $E=-\operatorname{tr}(\rho(t) \log \rho(t))$ as our entanglement measure, then we have

$$
\begin{aligned}
\Gamma(t) & =-\operatorname{tr}(\dot{\rho}(t) \log \rho(t))=-\sum_{n} \frac{d \lambda_{n}(t)}{d t} \log \lambda_{n}(t) \\
& =-2 \sum_{n, m=1}^{N} \sqrt{\lambda_{n}(t) \lambda_{m}(t)} \log \lambda_{n}(t) h_{n m}(t)
\end{aligned}
$$

where $h_{n m}(t)=\operatorname{Im}\left[\left\langle a_{n}(t)\left|\left\langle b_{n}(t)|H| a_{m}(t)\right\rangle\right| b_{m}(t)\right\rangle\right]$ is called entangling capability of the nonlocal Hamiltonian. In recent times, there have been various attempts to give bound on the entanglement rate. For example, upper bound on $\Gamma$ in the presence of local ancillas was proposed by Childs et al [9]. Wang and Sanders have shown that $\Gamma \leq \beta \approx 1.9123$ for any product Hamiltonian $H=X \otimes X$, where $X$ is a self-inverse Hamiltonian [10]. Bennett et al have shown that $\Gamma(H) \leq c d^{4}\|H\|$, where $d$ is number of Schmidt coefficients, and $c=O(1)$ [11]. Childs et al have shown that any two product Hamiltonians can simulate each other [12]. Bandyopadhyay and Lidar have investigated the effect of noise on the entangling capacities of two-qubit Hamiltonians [13]. Very recently, Lari et al have investigated the entanglement rate for two qubits, two qutrits and three qubits using a geometric measure of entanglement which holds for multiparticle systems [14]. However, in the literature there is no universal bound for entanglement rate. What we plan in this paper is to provide a universal bound on the time average of entanglement rate.

Before, giving a bound on the time average of entanglement rate, we will show that in some special cases, the entangling capability is directly related to the speed of the transportation of the entangled quantum system. Once we apply the non-local Hamiltonian, the state at a later time is given by $\mathrm{Eq}(1)$. Suppose, it is possible to perform time-dependent local unitary transformations and make the Schmidt basis $\left\{\left|a_{n}(t)\right\rangle\right\}$ and $\left\{\left|b_{n}(t)\right\rangle\right\}$ time independent. This 
can be imagined as the state of the entangled system in a bilocal rotating frame. Since local unitaries cannot change the entanglement, we can define a state

$$
\left|\Psi_{R}(t)\right\rangle=U^{\dagger}(t) \otimes V^{\dagger}(t)|\Psi(t)\rangle=\sum_{n=1}^{N} \sqrt{\lambda_{n}(t)}\left|a_{n}\right\rangle\left|b_{n}\right\rangle,
$$

where $\left|a_{n}(t)\right\rangle=U(t)\left|a_{n}\right\rangle$ and $\left|b_{n}(t)\right\rangle=V(t)\left|b_{n}\right\rangle$. Thus, at any given instant of time the entanglement content of $\left|\Psi_{R}(t)\right\rangle$ and $|\Psi(t)\rangle$ are the same, i.e., $E\left(\Psi_{R}(t)\right)=E(\Psi(t))$

Now, let us look at the (squared) speed at which the state $\left|\Psi_{R}(t)\right\rangle$ evolves in time. It is given by

$$
v^{2}=4 \frac{\Delta H^{2}}{\hbar^{2}}=\sum_{n} \frac{1}{\lambda_{n}(t)}\left(\frac{d \lambda_{n}(t)}{d t}\right)^{2} .
$$

For two-qubits with the general non-local Hamiltonian one has

$$
\frac{d p}{d t}=2 \sqrt{p(1-p)} \operatorname{Im}\left[\left\langle a_{0}\left|\left\langle b_{0}|H| a_{1}\right\rangle\right| b_{1}\right\rangle\right]
$$

where $\lambda_{1}=p$ and $\lambda_{2}=(1-p)$. Therefore, the speed of the system can be expressed as

$$
v^{2}=\frac{\dot{p}(t)^{2}}{p(t)(1-p(t))}=4 \operatorname{Im}\left[\left\langle a_{0}\left|\left\langle b_{0}|H| a_{1}\right\rangle\right| b_{1}\right\rangle\right]^{2} .
$$

Note that $h=\operatorname{Im}\left[\left\langle a_{0}\left|\left\langle b_{0}|H| a_{1}\right\rangle\right| b_{1}\right\rangle\right.$ and $h_{\max }$ is entangling capability of the non-local Hamiltonian. Therefore, from (17) we have $V=2 h$. Thus, $V_{\max }$, the maximum speed of the entangled system during quantum evolution is directly related to the entangling capability of two-qubit Hamiltonian. This shows that in a bilocal rotating frame the entangling capability is a geometric quantity.

Next, we will show that one can give a universal bound on the time average of entanglement for any initial state and general nonlocal Hamiltonians. Consider the evolution of a bipartite system $|\Psi(0)\rangle \rightarrow|\Psi(T)\rangle$ during an interval $[0, T]$. We define the time average of entanglement $\bar{\Gamma}$ as

$$
\bar{\Gamma}=\frac{1}{T} \int_{0}^{T} \Gamma(t) d t=-\frac{1}{T} \sum_{n} \int_{0}^{T} \log \lambda_{n}(t) d \lambda_{n}(t) .
$$

Note that during evolution of a composite system the change in the entanglement content can never exceed $\log N$, i.e., $\delta E=E(T)-E(0) \leq \log N$, where $N$ is number of Schmidt coefficients in the entangled state. This implies that $\bar{\Gamma} \leq \frac{\log N}{T}$. Now, using the geometric uncertainty relation for time and energy fluctuation, we find

$$
\bar{\Gamma} \leq 2 \log N \frac{\Delta H}{\hbar S_{0}}
$$

Thus, the maximal value of time average of entanglement rate depends on the maximum Schmidt rank, on the quantum fluctuation in the nonlocal Hamiltonian and inversely on the shortest geodesic path. For a given system, the time average of entanglement does not depend on the particular Hamiltonian, rather on the fluctuation in the Hamiltonian. 
There can be infinite number of nonlocal Hamiltonians, yet they all may give same average entanglement rate. For two qubits, starting with the initial state as given in (8), the maximal time average of entanglement is given by

$$
\bar{\Gamma}_{\max }=\frac{\left(\mu_{1}+\mu_{2}\right)}{S_{0}} \sqrt{1-4 p(1-p)} .
$$

It may be mentioned that it is not yet known whether the maximal entanglement and disentanglement rates of a nonlocal Hamiltonian are the same, i.e. if $\Gamma(H)=\Gamma(-H)$ ? We would like to point out that even though we do not know complete answer to the above question, our result suggests that the maximum attainable value for the time averaged entanglement rate and time averaged disentanglement rates are identical. The maximum of time average entanglement rate is given by $\bar{\Gamma}_{\max }(H)=2 \log N \frac{\Delta H}{\hbar S_{0}}$. Since it depends on the quantum mechanical uncertainty, we have $\Delta H=\Delta(-H)$ and because of the law of reciprocity of quantum mechanical probability $\left(S_{0}\right.$ is same during entangling and disentangling evolutions) we have $\bar{\Gamma}_{\max }(H)=\bar{\Gamma}_{\max }(-H)$. This is another non-trivial consequence of our geometric approach to the problem.

\section{MINIMUM TIME FOR COMPOSITE HAMILTONIAN}

Now, we ask what happens to the minimal time if we consider a time evolution under one Hamiltonian instead of another. In particular, if the later is build up from a composition of Hamiltonians then how does the minimum time behave. The answer to this question may be useful in quantum computing algorithms, where one tries to design sequence of unitary operators via the application of suitable Hamiltonians. It is known that if we have the ability to perform evolution under a Hamiltonian $H$ (say) and have the ability to apply unitaries $U_{k}$, it is possible to simulate evolution according to a composite Hamiltonian like $H^{\prime}=\sum_{k} \alpha_{k} U_{k} H U_{k}^{\dagger}$, where $\alpha_{k}$ 's are real numbers [16]. Under such an evolution we will have

$$
T \geq \frac{\hbar}{2 \Delta H^{\prime}} \cos ^{-1}\left|\left\langle\Psi(0)\left|U(T)^{\prime}\right| \Psi(0)\right\rangle\right|,
$$

where $U(T)=\exp \left(-i H^{\prime} T / \hbar\right)$ and $\Delta H^{\prime}$ is the quantum fluctuation in the composite Hamiltonian. Suppose, that the initial and the final states are fixed, i.e., we start with same initial state and want to reach a desired target state with the new Hamiltonian $H^{\prime}$. Then, the shortest path $S_{0}$ is again the same. Now, using the convexity property of quantum mechanical uncertainty [15], we have

$$
\Delta H^{\prime}=\Delta\left(\sum_{k} \alpha_{k} U_{k} H U_{k}^{\dagger}\right) \leq \sum_{k} \alpha_{k} \Delta\left(U_{k} H U_{k}^{\dagger}\right) .
$$

This tells us that mixing different Hamiltonians decreases the quantum mechanical uncer-

tainty. If we define $H_{k}=U_{k} H U_{k}^{\dagger}$, then the minimum time for the new Hamiltonian satisfies

$$
T \geq \frac{\hbar S_{0}}{2 \Delta H^{\prime}} \geq \frac{\hbar S_{0}}{2 \sum_{k} \alpha_{k} \Delta H_{k}} .
$$

This also gives a composition law for the minimum time for entangled brachistocrone problem under composite Hamiltonian. To illustrate this, consider a composite Hamiltonian 
$H^{\prime}$ that consists of two Hamiltonians $H_{1}$ and $H_{2}$. If we evolve the system only under $H_{1}$ then the minimum time to reach the target entangled state will be $T_{1_{\text {min }}}=\frac{\hbar S_{0}}{2 \Delta H_{1}}$. Similarly, if we evolve the system only under $H_{2}$ then the minimum time to reach the target entangled state will be $T_{2_{\text {min }}}=\frac{\hbar S_{0}}{2 \Delta H_{2}}$. Now, if we evolve the system under $H^{\prime}=\alpha_{1} H_{1}+\alpha_{2} H_{2}$ then the minimum time to reach the target entangled state will be

$$
T_{\min }=\frac{T_{\min 1} T_{\min 2}}{\alpha_{1} T_{\min 2}+\alpha_{2} T_{\min 1}} .
$$

To illustrate this composition law consider the initial state as given in (8). Now suppose, we apply the Hamiltonian $H_{1}=\mu_{1} \sigma_{1} \otimes \sigma_{1}$ and evolve the system for sometime to reach the target entangled state. In this case, the minimum time we need to wait is given by $T_{1_{\text {min }}}=\frac{\hbar S_{0}}{2 \mu_{1} \sqrt{1-4 p(1-p)}}$. If we apply instead $H_{2}=\mu_{2} \sigma_{2} \otimes \sigma_{2}$ and evolve the system to reach the target entangled state, then the minimum time we need to wait is given by $T_{2_{\min }}=$ $\frac{\hbar S_{0}}{2 \mu_{2} \sqrt{1-4 p(1-p)}}$. Now, we apply $H=\alpha_{1} H_{1}+\alpha_{2} H_{2}$ and evolve the system for sometime to reach the same target entangled state. In this case, the minimum time we need to wait is given by $T_{\text {min }}=\frac{\hbar S_{0}}{2\left(\alpha_{1} \mu_{1}+\alpha_{2} \mu_{2}\right) \sqrt{1-4 p(1-p)}}$. This later quantity indeed satisfies the composition law as given in (24). In fact, with a suitable controlled experiment, one can even test the composition law for the minimum time.

\section{CONCLUSION}

We have investigated the brachistochrone problem for entangled states. This answers the question that we have raised in the beginning, namely, given an arbitrary product state and general non-local Hamiltonian, what is the minimum time for creating a desired entangled state? We have shown that the minimum time depends inversely on the speed of the evolution of the quantum system. This is independent of the particular entanglement measure and holds even for multiparticle entangled systems. We have shown that for two qubits the presence of initial entanglement does help in minimising the waiting time. In particular, we find that if we are given an option of starting from a product state and an entangled state, and wants to reach the maximally entangled state, then it is better to start from some already initial entangled state. Because, in the later case the time required is less. We have shown that the time average of entanglement rate depends on the Schmidt rank and on the fluctuation in the non-local Hamiltonian. We have also shown that in a bilocal rotating frame the entangling capability is also directly related to the speed of the evolution of the entangled states. This shows that the entangling capability of a non-local Hamiltonian is actually a geometric quantity. Furthermore, we have proved a composition law for the minimum time when the system evolves under composite Hamiltonians. We hope that these findings will be of interest in understanding the entanglement rate and entangling capabilities of non-local Hamiltonians and in real experimental situations. In future, it will be interesting to see if all these findings also hold for qudits and multiparticle systems.

[1] D. P. DiVincenzo and C. H. Bennett, Natute (London) 404, 247 (2000).

[2] C. A. Sackett et al, Nature (London) 404, 256 (2000). 
[3] B. Julsgaard et al, Nature (London) 413, 400 (2001).

[4] A. J. Berkley et al Science 3000, 1548 (2003).

[5] W. Dur, G. Vidal, J. I. Cirac, N. Linden, and S. Popescu, Phys. Rev. Lett. 87137901 (2001).

[6] J. Anandan and Y. Aharonov, Phys. Rev. Lett (1990).

[7] A. K. Pati, Phys. Lett. A (1991).

[8] A. K. Pati, Phys. Rev. A 52, 2576 (1995).

[9] A. M. Childs, D. W. Leung, F. Verstraete, and G. Vidal, Quantum Inf. Comput. 3, 97 (2003).

[10] X. Wang and B. C. Sanders, Phys. Rev. A 68, 014301 (2003).

[11] C. H. Bennett, A. W. Harrow, D. W. Leung, and J. A. Smolin, IEEE Trans. Inf. Theory, 49, 1895 (2003).

[12] A. M. Childs, D. W. Leung, and G. Vidal, IEEE Trans. Inf. Theory, 50, 1189 (2004).

[13] S. Bandyopadhyay and D. A. Lidar, Phys. Rev. A 70, 0101301 (2004).

[14] B. Lari, A. S. M. Hassan and P. Joag, Phys. Rev. A 80, 062305 (2009).

[15] A. K. Pati and P. K. Sahu, Phys. Lett. A 367, 177 (2007).

[16] M. A. Nielsen, M. J. Bremner, J. L. Dodd, A. M. Childs, C. M. Dawson, Phys. Rev. A 66, 022317 (2002). 